This is the accepted manuscript of the article, which has been published in 11th ACM Symposium on Eye Tracking Research and Applications, ETRA 2019. 2019, a85. http://dx.doi.org/10.1145/3317959.3321489

\title{
Eye gaze and head gaze in collaborative games
}

\author{
Oleg Špakov \\ Tampere University \\ Tampere, Finland \\ Tiia Viitanen \\ Tampere University of Applied \\ Sciences \\ Tampere, Finland
}

\author{
Howell Istance \\ Tampere University \\ Tampere, Finland
}

\author{
Kari-Jouko Räihä \\ Tampere University \\ Tampere, Finland
}

\author{
Harri Siirtola \\ Tampere University \\ Tampere, Finland
}

\begin{abstract}
We present an investigation of sharing the focus of visual attention between two players in a collaborative game, so that where one player was looking was visible to the other. The difference between using head-gaze and eye-gaze to estimate the point of regard was studied, the motive being that recording head-gaze is easier and cheaper than eye-gaze. Two experiments are reported, the first investigates the effect of a high immersion presentation of the game in VR Head Mounted Display compared with a lower immersion desktop presentation. The second examines the high immersion condition in more detail. The studies show that in spite of there being many factors that could affect the outcome of a relatively short period of game play, sharing eye-gaze in the high immersion condition produces shorter overall durations and better subjective ratings of team work than does sharing head-gaze. This difference is not apparent in the low immersion condition. The findings are a good argument for exploiting the opportunities for including and using eye tracking within head mounted displays in the context of collaborative games.
\end{abstract}

\section{CCS CONCEPTS}

• Applied computing $\rightarrow$ Computer games; $\bullet$ Human-centered computing $\rightarrow$ Collaborative and social computing systems and tools; Pointing.

\section{KEYWORDS}

VR, gaze sharing, collaborative games, gaze in teamwork

\section{INTRODUCTION}

As eye tracking technology has become more accessible and less expensive in recent years, so research interest into how eye gaze data can be usefully shared in different contexts has grown. Visual search tasks where two [Brennan et al. 2008] or more [Siirtola et al. 2019] people work together to locate targets has been shown to be more efficient by making the gaze positions of the other searchers visible. This can lead to strategies for sharing the work to evolve without these needing to be explicitly agreed in advance.

Remote collaboration has been shown to be significantly improved in the context of a remote expert helping someone less expert to solve a work-related problem, for example in the context of on-site maintenance. Gupta et al. showed a significant improvement in performance if the gaze position of a local person was shared with the remote person in addition to sharing the view of the local person obtained from a head mounted camera [Gupta et al. 2016]. Additionally, the provision of eye position information and the use a pointer significantly improved the co-presence felt between the two workers.

Another avenue of work where the inclusion of eye tracking technology is set to make a very considerable impact is in the use of VR-headsets ${ }^{1}$. In addition to enabling foveated rendering and foveated displays, eye gaze position can be used to convey intent and objects of interest between different people in the same virtual space. This could be used for many applications, such as training control room operatives using virtual representations of a control room, or for meetings to review architectural projects.

An area that unites these directions is the design of collaborative games, where the players need to work together to undertake a joint mission. Such games could have an educational purpose, such as teaching teamwork skills [Hamalainen 2008], or could have entertainment as the primary purpose. It is of interest to know the extent to which sharing eye gaze between players contributes to game-related performance and teamwork, compared with sharing only the direction that a person is facing (head gaze). It is also of interest to establish whether such benefits depend on the level of immersion afforded by the display system, such as a VR head-mounted display (HMD) compared with a desktop screen and conventional input devices.

There are two research questions that the work reported in this paper seeks to answer. The first is what is the added value of eye gaze over head gaze in the context of collaborative games. There

\footnotetext{
${ }^{1}$ At https://www.roadtovr.com/why-eye-tracking-is-a-game-changer-for-vr-headsetsvirtual-reality/, accessed January 2019
} 
are two principal uses of these gaze modes under consideration here. One is to point at an object in the game, and the other is to indicate to the other player what the player is currently looking at. The second research question asks whether the added value of eye gaze over head gaze is affected by the level of immersion both players experience while playing the game. The motivation for the study is to question whether the additional expense and overhead of eye tracking players in an immersive virtual environment is worthwhile in the context of collaborative game play.

In the first of two experiments, the difference between two people playing a collaborative game using eye gaze and head gaze is compared between a more immersive condition and a less immersive condition. This uses a between-participants design. In the second experiment, the difference between eye-gaze and head-gaze is studied in the more immersive condition only using a withinparticipants design.

\section{BACKGROUND}

There are several areas of related work that inform this work. There is the use of eye-gaze for pointing at and selecting objects in a VR context. There is the use of gaze to interact with games for both command-based interaction and non-command-based interaction. There is also the question of whether sharing eye-gaze between players can assist in collaborative work and in playing team games, and how this aspect of collaborative games can be evaluated.

Early comparisons of eye pointing and head pointing in the context of assistive devices showed eye pointing performance generally to be poorer especially for smaller targets [Bates and Istance 2003]. Here an on-screen pointer was moved by head or by eye to select interface objects in a standard word processor using a hand-held micro-switch in both cases. This was the result of rapid but inaccurate eye pointing compared with slower but more accurate head pointing. The advent of VR HMDs equipped with eye tracking capability mean that these modalities can be combined. The selection cursor may be locked to the centre of the field of view and controlled entirely by head movement (head-gaze), or it may be located at the gaze position within the field of view (eye-gaze). Moving the pointer in the latter condition can be achieved by a combination of eye and head movements.

In a Fitt's Law ISO 9241-9 selection task, using a FOVE HMD, head pointing outperformed both eye pointing and the combination of eye and head pointing [Qian and Teather 2017]. The authors speculated that poor precision of the integrated eye tracking system may have been responsible for this. Eye-gaze and head-gaze selection were compared for different simulated fields of view in VR and AR systems [Blattgerste et al. 2018] using a HTC Vive and integrated eye tracking from SMI. They found that pointing performance with eye-gaze was significantly better than with head-gaze and that this advantage was more pronounced with larger fields of view. The benefits of eye-gaze require a well calibrated and accurate eye tracker.

Murray et al. [Murray et al. 2007] studied a person's ability in an immersive virtual environment to judge where an avatar representing another person was looking. They showed a significant benefit of integrating the display of an avatar's gaze direction with the head direction. This improved the ability of a person to determine which object in an immersive virtual environment the avatar was looking at, which were located in a large $3 \times 3$ grid in front of it. Eye tracking and head tracking data previously recorded from a human participant looking at objects was used to animate the display of the avatar. In addition to gaze direction, they also showed a benefit for showing the eye vergence of the avatar in identifying objects at different distances.

The coupling between eye movements of people working together has been studied as a predictor of success in computer supported collaborative work [Chanel et al. 2013]. This has been extended to displaying the location of the collaborating person's gaze position in the view that a person has of a remote work space. Displaying both the collaborator's pointer and gaze position enabled participants to perform the task being studied significantly faster [Gupta et al. 2016]. It also enabled conversations to be more efficient, and helped participants to feel more connected.

Dual eye tracking has been used to study the interaction between novice and expert players in a collaborative version of Tetris [Jermann et al. 2010] and how playing styles change according to the respective experience levels of the two players. However, they did not show one player the gaze position of the other in real time as this could disturb game play. Sharing real-time gaze position of one player to the other in a game of checkers was shown to help the gaze viewing player to predict the moves of the gaze tracked player [Kumar et al. 2018]. Eye tracking of expert players has also been used in a system for automatic annotation and analysis of moves in chess [Küchelmann et al. 2017].

Sharing eye gaze between remotely located game players can be thought of as contributing to physiological linkage and an increased sense of social presence. The latter is thought to have a number of dimensions: co-presence, a sense of being in touch with another person; psychological involvement; and behavioural involvement [Biocca et al. 2003; Ekman et al. 2012]. This is of significant interest, not least in gaming, of designing interfaces between people in remote locations to increase the sense of social presence between them. The value of displaying an opponent's eye-gaze position in the view a player has of a game in online competitive game play has been studied [Lankes et al. 2016], and has been found to increase awareness and engagement between the two players.

\section{DESIGN OF A COLLABORATIVE GAME}

A multi-player game was designed and built in Unity. This could be displayed on the screen of a laptop, use data from a remote eye tracker and a keyboard and mouse (Desktop version). The same game could also be displayed in a head mounted display, use data from an integrated eye tracker and a hand-held controller (VRHMD version). The game supported pointing by both head gaze and eye gaze.

As the intention was to test numbers of pairs of students playing the game together, the game had to be quick to learn. It also had to be quick to complete (taken to mean about 10 minutes after initial familiarization was completed). We required there to be a pointing element (without a selection action) that could be achieved using head or eye gaze. We wanted the two players to collaborate and communicate with each other. A play balance was needed so that a pair of students with a moderate to high amount of game 


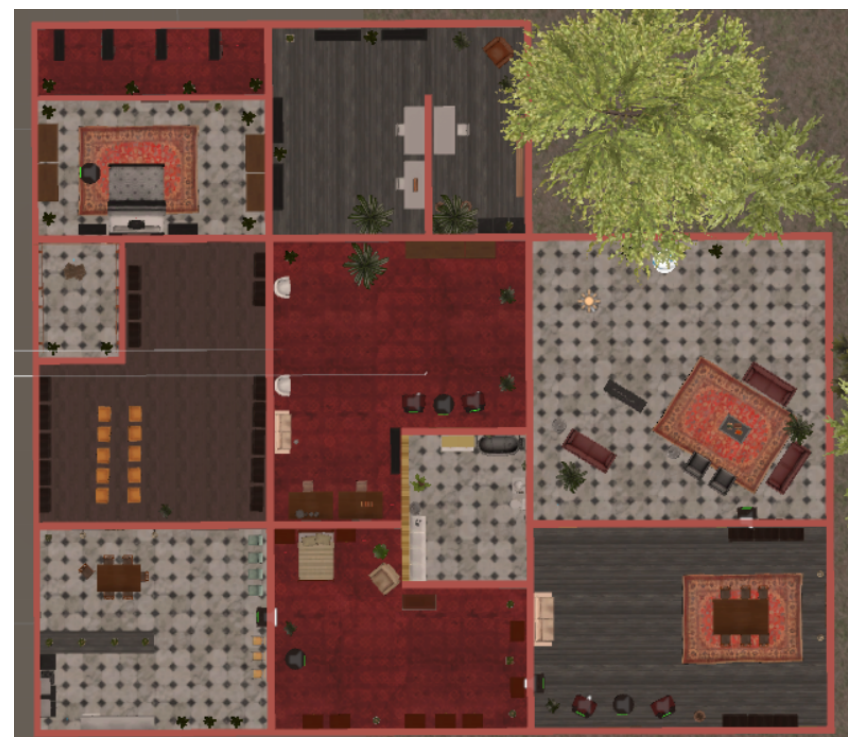

Figure 1: The layout of the rooms in the house, the game began in the room on the middle right of the figure

play experience, but no experience of VR, would find the game engaging and motivating, and could complete the game within the allotted time. A search task was chosen that required both players to navigate through an unfamiliar space and collect items, whilst being under the threat of attack from non-player characters.

The theme of the game was to explore a darkened empty house to find keys located in different rooms. All rooms were locked initially and as a key was found, the door to the next room was opened automatically. That room was either directly accessible from the current room, or it could be necessary to backtrack one or two rooms to find the newly opened door. There were 11 rooms in the house and the layout is shown in Figure 1.

The keys were hanging in mid-air in their respective rooms and were collected by colliding with them. There was a total of 10 keys, and the final key opened the door to the garden outside. This door was in the first room where the game started (on the right of Figure 1) and players were told to go there and leave the house as quickly as possible when the $10^{\text {th }}$ key had been collected. Each player had a torch, which illuminated an area in front of the player. The direction of the torch was either fixed in the centre of the viewport (headgaze) or was fixed to the eye gaze position within the viewport (eye-gaze).

Figure 2 shows one player's view while in the starting room. The player's torch is in centre of the viewport. The other player is visible in front of this player, and that player's torch is shining onto the left side of the room. The key to leave this room is just visible in the centre of the other player's torch light. The number of keys collected so far by both players are shown on the top left of the display.

In the Desktop version of the game, the field of view was rotated with the mouse, and the WASD keys were used to move and the SPACE key to jump. In the VR-HMD version of the game, a touch-pad located on a hand-held controller was used to move, and functioned as a joystick without the need to press the pad. A trigger located on the backside of the controller was used to jump. The field of view was rotated by turning the head, and players were seated to prevent becoming tangled in the cable connecting the HMD to the computer.

The rooms contained furniture. Some pieces of furniture (a grandfather clock, a table and a chair) could turn into monsters that approached players when they were in range. When a monster was activated for the first time, it made a 'roaring' sound (each type of monster made a unique sound). A player lost health when monsters collided with them. The amount of health damage caused by a collision, the movement speed and sensitivity range were dependent on the type of monster. If the player did not move during the monster attack, it usually took from 5 to 8 seconds to lose health from $100 \%$ to $0 \%$. In this case, players respawned immediately but back in the starting room.

Players could disarm and stop a monster by pointing the light torch at the monster's face. When a monster's health was lost completely, it was deactivated for 10 seconds and remained at that location before regaining health and moving again. Monsters would stop moving when both players were out of range.

\section{EVALUATING EYE-GAZE IN COLLABORATIVE GAME PLAY}

\subsection{Experiment 1 - Impact of level of immersion on eye-gaze and head gaze differences}

As described in Section 2, there is not a clear picture of whether eye-gaze is better than head-gaze. Factors including the application context, the quality of the eye tracking data, and possibly the field of view may all influence the relative merits for the two modes. Within the context of collaborative game play, we wished to examine the impact of the level of immersion on these relative merits. By immersion, we refer to the extent to which the display and input devices affect a person's sense of presence in the game. [Jennett et al. 2008]. The high immersion condition was the VR-HMD version and the low immersion condition was the Desktop version (see Section 3)

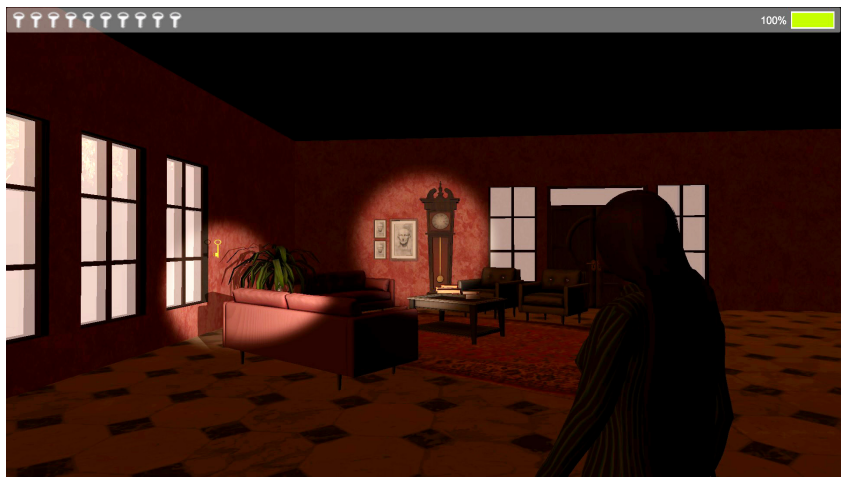

Figure 2: A screenshot from the game 


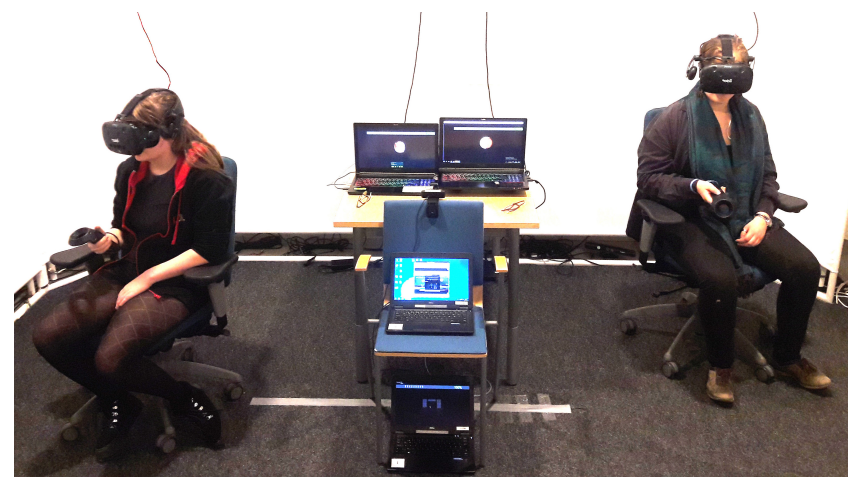

Figure 3: VR setup: two participants are playing the game while wearing HTC VIVE headsets

4.1.1 Participants. We recruited 40 participants (31 males, 6 females, 3 others) from 20 to 30 years old (mean=23). Most of the participants (38) reported that they played games several times a week (13) or daily (25). Also, most of them were asked to sign up for the study in pairs, so 30 participants knew their partner quite well or very well, and only 3 participants reported they did not know or had never met the partner. Previous experience using HMDs varied, five players had no experience in using HMDs, seven had tried it once or twice, and six players had used them more frequently. The pairs were self-selecting and no attempt was made to match game experience or expertise between the two players.

4.1.2 Equipment setup. In the Desktop version, the game was installed onto two DELL laptops (Intel i5-5300U 2.3 GHz, 8 GB RAM, Intel HD Graphics 5500). A myGaze eye tracker produced by Visual Interaction and a conventional mouse were connected to each of the gaming laptops. One more laptop was used to run the game server. In the VR-HMD version, there were two HTC VIVE headsets with Tobii eye tracker built-in and a hand-held controller connected to it remotely. The overview of this setup is shown in Figure 3. Each headset was connected to a separate MSI laptop (Intel i7-7700HQ 2.8GHz, 16 GB RAM, NVidia GeForce GTX 1060) by a long cable; the cable was hanging from a ceiling allowing participants to freely rotate in space and move within a small area.

4.1.3 Design and Procedure. Twenty pairs of participants played the game. All were games design undergraduates from a local university. Ten played the Desktop version and ten played the VR-HMD version. Within these groups, five pairs used head-gaze and five used eye-gaze to control the torch. Both players were in the same physical space, They could talk to each other but not see each other. In the Desktop condition players sat opposite each other with a high cardboard screen between them.

The pair of participants were first asked to fill in a consent form and background data questionnaire. The objective of the game was explained, followed by a description of the way to control movement using either the keyboard and mouse or the hand held controllers. The participants using the VR-HMD version were warned about the possibility of motion sickness. They were asked to say if they felt nauseous, and told that the game would be stopped. Players were not told about the different conditions in the study, nor which
Table 1: Statements relating to teamwork after [Gupta et al. 2016]

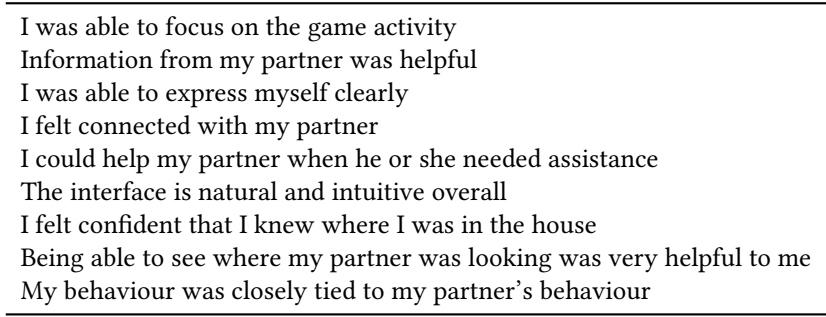

condition they were in. They were reminded that they could talk to each other throughout the game. No other guidance about how they might work together during the game was given.

After the experiment was over, a presentation of the experiment and results was made to the whole group of participants.

The pairs using eye-gaze then went through a simple 5-point calibration procedure. The game was started and the first room was treated as a training room. The players then were asked to practice to rotate and move around the room to get used to WASD keys and HTC controllers. There was a monster in the room but it caused no damage to players' health. They were asked to practice disarming this monster. After the first key was captured, the door to the next room was opened automatically. The game started when the first player left the training room and the experimenter did not talk to the players until the game was over.

4.1.4 Data collected. All actions of players and monsters were logged. The log files also contained events of light beams entering and leaving monsters, player respawns, monster activation and deactivation, keys collected and doors passed through. The content of both players' screens and their voices were captured by a single web-camera. Two monitors showing each screen were placed side by side and filmed, as shown in Figure 3.

When the game was over, each player was asked to individually rate their agreement with nine statements relating to teamwork.

Table 1 lists the statements used to rate teamwork. A 5-point Likert scale was used to record level of agreement, ranging from 'very much disagree' to 'very much agree'. The statements were those used in Gupta et al.'s [Gupta et al. 2016] study of the role of eye gaze in collaborative work, with modifications to reflect the nature of the game activity.

Following this, the players were interviewed together about teamwork and how it had evolved between them during the game. They were asked about ease of controlling the avatar and the lighttorch.

\subsection{Results - Experiment 1}

4.2.1 Game Duration. The total time taken to complete the game can be used as a coarse indicator of how efficiently the 2 players could complete the task of finding the keys and leaving the house. Figure 4 shows the mean total durations in the 2 mode conditions and the 2 immersion conditions. The duration is defined as the time from the first player leaving the first (training) room until both players have left the house and the game is over. 


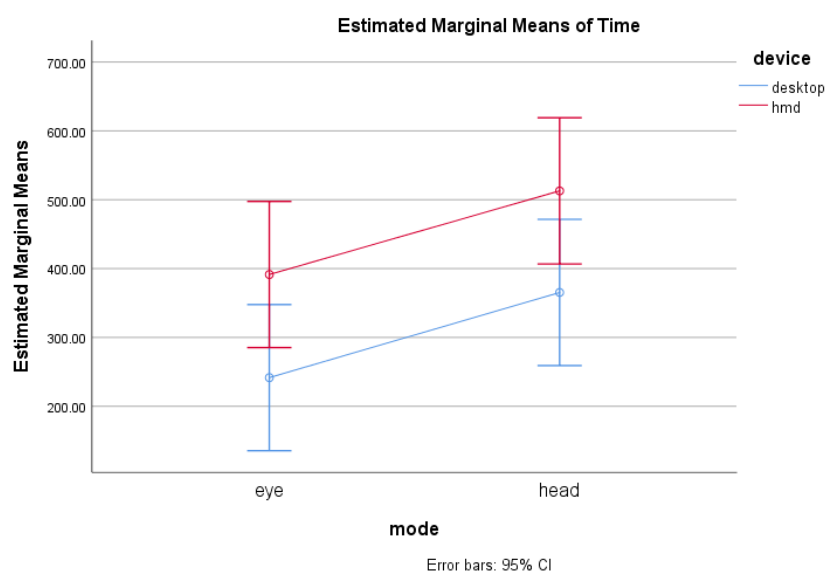

Figure 4: Total durations of games shown by mode (eye gaze and head gaze) and immersion condition (Desktop and VRHMD)

There are significant main effects for immersion condition (Desktop and VR-HMD) $(\mathrm{p}=0.009)$ and for mode (eye-gaze and head-gaze) $(p=0.026)$. There is no significant interaction effect. Leverne's tests of equality of the error variances of duration across groups show no significant outcomes. The Shapiro-Wilk test shows the time data to be normally distributed when partitioned by immersion condition and by mode.

The game was completed significantly more quickly by pairs using eye-gaze compared with head gaze. It was completed significantly more quickly in the Desktop version compared with the VR-HMD version. One reason for this may be that participants were less familiar with the use of the hand-held controllers compared with the keyboard and mouse.

4.2.2 Specific collaboration metrics. Collaborative activities can only be partly inferred from the logged data. All events when both players were disarming the same monster were counted as evidence of collaboration. Collaboration was also observed in cases when one player was searching for a key and the other was disarming a monster. This behaviour does not necessarily imply collaboration, as the player 'hunting' a monster could do it for reasons not connected with defending another player. However, both players located in the same room usually meant that neither had been left behind. An exception was where one player had been respawned in the first room, and was rejoining the other player. The computed values reveal that both players were in the same room for approximately same share of the game duration (63-69\%) in all conditions except for the VR-HMD condition with head-gaze (36.5\%). If only the intervals are counted when players are in the room where a key is located, then this measure is higher in eye-gaze mode for both immersion conditions: $27.6 \%$ vs $37.5 \%$ in the Desktop version, and $6.9 \%$ vs $31.7 \%$ in the VR-HMD version (see Figure 5, right). The players were both disarming the same monster for $0.5 \%-3.3 \%$ of the time they spent in the game, but without any clear differences between conditions (see Figure 5, left).
4.2.3 Subjective ratings of Teamwork. All statements in the questionnaire were favourable towards the psychological object (i.e. teamwork), and responses were scored from 0 ('Very Much Disagree') to 4 ('Very Much Agree'). In accordance with Likert's method of summated ratings [Oppenheim 2000], the ratings for the nine statements were summed for each participant to provide a score that ranged from 0 to 36 .

Table 2 shows the teamwork scores and the corresponding ranks and rank sums for head-gaze and eye-gaze in both the Desktop condition and the VR-HMD condition. The Wilcoxon's Rank-Sum Test was used to compare head-gaze and eye-gaze within each of the immersion conditions respectively. Scores are ranked in ascending order, so the lower the scores in a condition, the lower the ranks and the lower the rank sum.

In the VR-HMD condition, eye-gaze produces significantly higher teamwork scores than does head-gaze. The rank sum in the eye gaze condition is 133, while in the head gaze condition, the rank sum is 77 . The probability of obtaining a rank sum as high as 133 from the pooled scores from 2 samples where N1 = 10 and N2 = 10 under the null hypothesis is 0.025 for a 1-tailed test. This corresponds to 0.05 for a 2-tailed test, which is appropriate for use here. In the Desktop condition, the head gaze condition produces higher teamwork scores than eye gaze, although the difference here is not significant. In the condition with the higher level of immersion, eye-gaze gives rise to significantly higher subjective scores of teamwork than head-gaze.

4.2.4 Post-trial interviews with both players in a pair. In the postgame interviews, the comments made about how well collaboration worked were fairly similar between the pairs in the eye-gaze and the head-gaze modes in both the Desktop and the VR-HMD conditions. In the eye-gaze mode in the VR-HMD condition, participants however were clearer about the basis upon which collaboration was based than in the other conditions. This was usually on the basis that while one player looked for a key, the other kept monsters away from that player. When asked about the ease of controlling the torch, the responses were very similar between eye-gaze and head-gaze in both the VR-HMD and the Desktop conditions.

Roughly half of the players (equally in all conditions) mentioned that it was useful to observe the partner's light-beam.

\section{Experiment 1}

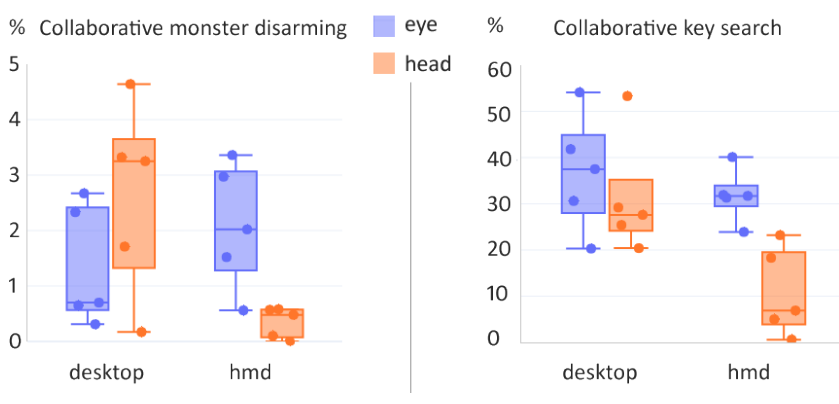

Figure 5: Share of game duration (left) both players spent on disarming a monster together, and (right) both players stayed in the same room when there was a key 
Table 2: Rank sums of teamwork scores for head-gaze and eye-gaze for Desktop and VR-HMD conditions. In VR-HMD, eye-gaze gives significantly highr ratings than head-gaze ( $p$ $=0.05$ ), in Desktop, no significant difference

\begin{tabular}{cccccccc}
\hline \multicolumn{4}{c}{ Desktop } & \multicolumn{4}{c}{ HMD } \\
\hline \multicolumn{2}{c}{ Head } & \multicolumn{2}{c}{ Eye } & \multicolumn{2}{c}{ Head } & \multicolumn{2}{c}{ Eye } \\
\hline score & rank & score & rank & score & rank & score & rank \\
\hline 29 & 13 & 19 & 1 & 17 & 2 & 25 & 9 \\
30 & 15.5 & 32 & 19.5 & 27 & 14 & 26 & 12 \\
23 & 3.5 & 26 & 6 & 18 & 3 & 30 & 17 \\
24 & 5 & 23 & 3.5 & 22 & 4 & 16 & 1 \\
31 & 17.5 & 28 & 9.5 & 25 & 9 & 30 & 17 \\
30 & 15.5 & 29 & 13 & 23 & 5 & 25 & 9 \\
28 & 9.5 & 32 & 19.5 & 24 & 6.5 & 32 & 19.5 \\
21 & 2 & 28 & 9.5 & 28 & 15 & 30 & 17 \\
29 & 13 & 28 & 9.5 & 26 & 12 & 32 & 19.5 \\
31 & 17.5 & 27 & 7 & 24 & 6.5 & 26 & 12 \\
\hline \multicolumn{9}{c}{112} & 98 & \multicolumn{7}{c}{} & 133 \\
\hline
\end{tabular}

The participants had a number of ideas of how the game could be improved. Most often, they mentioned that monsters should be more dangerous (e.g., faster) (6), the keys could be hidden rather than being visible at distance (3) and the game could have more sound effects (3).

\subsection{Experiment 2 - Within-participants comparison in high immersion condition}

The first experiment showed that eye-gaze produced better ratings of teamwork and better overall performance than head-gaze in the higher immersion condition. To explore this further, a second experiment was conducted. A new set of participants played the game twice, with head-gaze and eye-gaze in the VR-HMD condition only.

4.3.1 Participants. We recruited 24 participants (17 males, $7 \mathrm{fe}-$ males) from 19 to 44 years old $($ mean=25). All were computer science undergraduates from the university. Most of the participants (17) reported that they are playing games several times a week (6) or daily (11). Only 3 participants reported they play games rarely or very infrequently. Only 6 participants knew their partner quite well or very well, and most of the participants (14) reported they do not know or had not met their partner previously. Most participants had very limited or no previous experience of using HMDs.

4.3.2 Revised version of game. In response to feedback from participants in Experiment 1, each monster was provided with a health bar to show the time remaining for staring at the monster's face to 'kill' it. Additionally, feedback was added to the monster to show when it was taking damage by being stared at, and feedback was added to the scene to show when a monster was causing a player health damage. Before the second experiment, a number of changes were made to the game based on observations and participant feedback from Experiment 1. It had been possible to look for and collect keys with little or no communication or teamwork between the two players, although this did not happen often. To increase the need for collaboration, the 10 keys were divided into 5 red and 5 blue keys. Each player could only collect keys of a particular colour and the player's 'own' colour had to be discovered in the first room. In the second experiment, each dyad played the game twice, once using head-gaze and once using eye-gaze. The locations of the sets of keys were different in each room for the two runs. The sets of locations were chosen to be equally easy or difficult. Also the need for back tracking was removed, so each key opened the door to next room from the room in which it was found. While this made the game easier to complete, we expected it would increase collaboration, as players were more likely to work together if they were in the same room. In the initial version where backtracking was sometimes required, players could split up and search separately for the room that had been opened by the most recent key found.

4.3.3 Design and Procedure. This experiment used a 2x2 crossover design. Each pair played the game twice in VR-HMD mode only (referred to here as Run 1 and Run 2). Half of the pairs had the sequence head-gaze control of the torch followed by eye-gaze control of the torch. The other half of the pairs had the sequence eye-gaze control followed by head-gaze control. One of the pairs in this latter sequence did not complete the head-gaze run as one of the participants reported symptoms of motion sickness and the trial was discontinued.

The procedure was similar to the one used in Experiment 1. The first room in Run 1 was used for training. However, as the participants played the game twice, each participant filled in the teamwork assessment questionnaire twice, one after each run. There was a short break between the runs. A joint interview was held after the second run.

\subsection{Results - Experiment 2}

4.4.1 Game Duration. Learning how to play the game had an effect on the overall durations. All second runs were shorter than the first runs. The assumptions of normality and equivalence of variance between conditions were not met, so a non-parametric approach to the analysis was taken instead [Putt and Chinchilli 2004]. The medians of durations are shown in Figure 6 together with $75^{\text {th }}$ and $25^{\text {th }}$ percentiles. The basis of the comparison between head-gaze and eye-gaze are the differences in the durations of the runs in the two conditions by each pair of players. These differences are ranked in ascending order. The sum of the ranks from the sequence Eye-then-Head is compared with that from the sequence Headthen-Eye using Wilcoxon's Rank Sum test. This shows that there is no significant difference between these, and so there is no difference is durations attributable to the use of eye-gaze and head-gaze.

4.4.2 Specific collaboration metrics. As with Experiment 1, two measures of probable collaboration were used. One is the amount of time as a proportion of the total duration that both players worked together to disarm the same monster. The other was the amount of time that the players were in the same room as the key being looked for. These two measures for the two runs are shown in Figure 7.

In the eye-gaze mode, the median proportion of game duration that both players were pointing the torch at the same monster was approximately the same in the first and second runs. In the head-gaze mode this decreases in the second run although these proportions of the total duration are small. Those pairs who used 


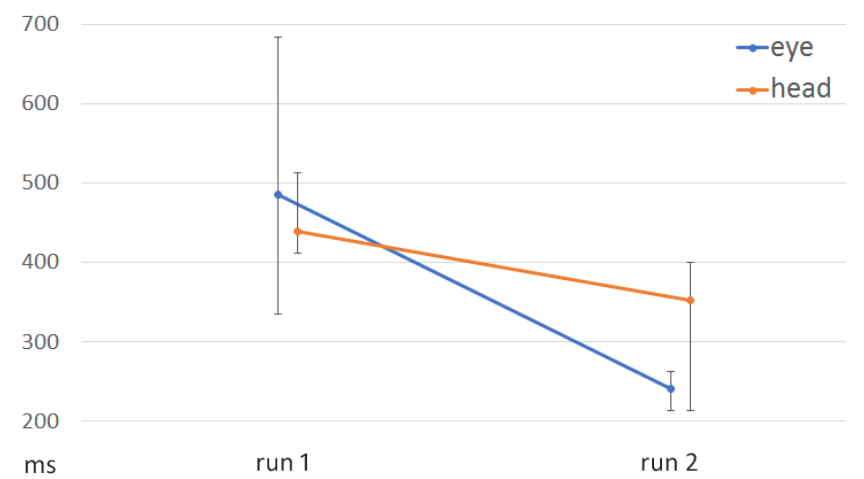

Figure 6: Medians of total durations of games shown by mode (eye gaze and head gaze) and order (Run 1 and Run 2). Error bars are 75 and 25 percentiles.

\section{Experiment 2}

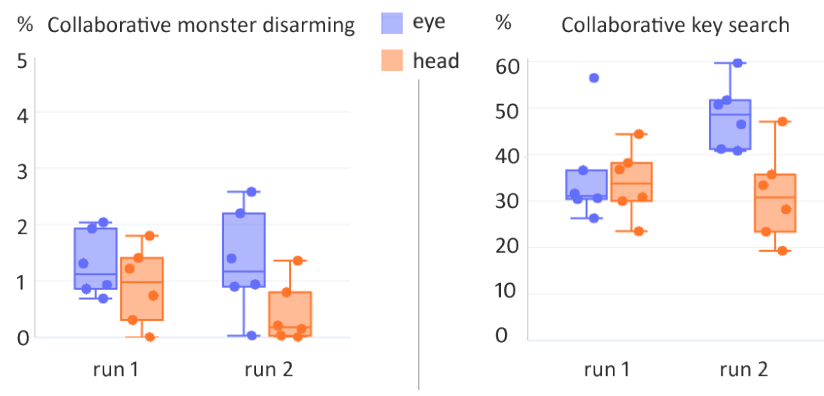

Figure 7: Share of game duration (left) both players spent on disarming a monster together, and (right) both players stayed in the same room when there was a key. Data is shown for both Run 1 and Run 2

eye-gaze in the second run spent longer disarming monsters together than they had in the first run with head-gaze $(+0.4 \%$, see the left hand side of Figure 8 ). Those pairs who began with eyegaze in the first run spent longer disarming monsters together than they did in the second run while using head-gaze (a decrease of $0.9 \%$ ). The absolute difference was small but significant although the relative changes were $44 \%$ and $-78 \%$ correspondingly.

The other measure of collaboration was the time spent together in the same room as the key being looked for (right hand side of Figure 7). On the first run in both sequences, overall these are very similar, while in the second run, the proportion of the total duration for those pairs using eye-gaze increases sharply (the mean duration increasing from around $30 \%$ to around 50\%). Within pairs, those with head-gaze in the second run spent less time together in the same room as the key being looked for compared with the first run (an absolute decrease of 2.3\%, a relative change of -7.5\%) (see Figure 8 , right). Those pairs with eye-gaze in the second run increased the proportion of the duration that they spent together in 'current key' room by an average of $14.5 \%$, a relative change of $+43 \%$. These differences were significant.
Table 3: Ranks and rank sums for differences in teamwork scores for 1st sequence (Eye-then-Head) and for 2nd sequence ( Head-then-Eye)

\begin{tabular}{cccccccc}
\hline \multicolumn{3}{c}{ 1st Sequence $(\mathrm{E}-\mathrm{H})$} & \multicolumn{4}{c}{ 2nd Sequence $(\mathrm{H}-\mathrm{E})$} \\
\hline 1st E & 2nd H & $\mathrm{D}$ & Rank & 1st H & 2nd E & $\mathrm{D}$ & Rank \\
\hline 24 & n/a & n/a & & 31 & 35 & -4 & 1.5 \\
22 & n/a & n/a & & 27 & 27 & 0 & 5 \\
29 & 25 & 4 & 15.5 & 27 & 24 & 3 & 11 \\
25 & 25 & 0 & 5 & 29 & 26 & 3 & 11 \\
34 & 24 & 10 & 22 & 27 & 27 & 0 & 5 \\
28 & 24 & 4 & 15.5 & 33 & 29 & 4 & 15.5 \\
34 & 29 & 5 & 18.5 & 28 & 22 & 6 & 20.5 \\
31 & 28 & 3 & 11 & 36 & 30 & 6 & 20.5 \\
32 & 30 & 2 & 7.5 & 12 & 16 & -4 & 1.5 \\
24 & 19 & 5 & 18.5 & 26 & 23 & 3 & 11 \\
27 & 28 & -1 & 3 & 33 & 29 & 4 & 15.5 \\
30 & 27 & 3 & 11 & 23 & 21 & 2 & 7.5 \\
\hline \multicolumn{9}{c}{127.5} & & & & 125.5 \\
\hline
\end{tabular}

4.4.3 Subjective ratings of teamwork. Using the non-parametric approach to the analysis of the $2 \times 2$ crossover design as described earlier, the differences in each player's rating of teamwork were calculated. These were partitioned into players belonging to the pairs in each sequence (Table 3). The differences are ranked across both sequences. The ranks are then summed within each sequence. If one gaze mode gave rise to higher teamwork scores than the other, then positive differences in one sequence would be mirrored by negative differences in the other sequence. Ranking all of the differences, both positive and negative, and summing these within each sequence would lead to differences in the rank sum of the two sequences. As can be seen from Table 3, the rank sums are very similar and there is no evidence of any significant differences in the teamwork scores between the gaze modes.

4.4.4 Post-trial interviews with both players in a pair. Eight pairs out of 11 reported that they had collaborated well in the game, and most reported some specific division of work. Nine participants said that they had experienced problems learning to use the handcontroller in the first run, but all of these had become familiar with using it by the second run. Half of the participants said that stopping monsters was the most challenging part of the game. Only one participant reported it was somewhat hard to memorize the layout of the house.

On the question of the ease of control of the torch, only one participant mentioned that in one run it was rather difficult to control the light-beam. In total, 14 participants did not notice there was a difference in the way light beam was controlled between the runs. From those who had noticed, only one participant claimed that it was easier to control the light beam with the head; four participants claimed the opposite, and the rest did not express any preferences.

\section{DISCUSSION}

The main contribution of this work has been to demonstrate the added value of sharing eye-gaze position rather than head-gaze 
position on teamwork in collaborative games. This is where the presentation of the game uses a high immersion head mounted display. The same advantage of eye-gaze over head-gaze is not apparent in the low immersion condition. One might reasonably expect that the difference between head-gaze and eye-gaze would be small in comparison with other factors. For some participants, it was one of their first experiences of game playing using an HMD. In some cases, participants knew the person they were playing with well and in others they did not know each other at all. In all cases the period of game play was short and the game was not previously known to the players. Nevertheless, there were significant differences between the eye-gaze and head-gaze conditions in Experiment 1 and to a lesser extent in Experiment 2. In the eye-gaze control condition, overall durations were shorter than in the head-gaze control condition in both the more familiar desktop environment and the less familiar VR environment. Ratings of teamwork were higher from participants using eye-gaze in the VR environment than from those using head-gaze. There was no similar difference however in the desktop condition. This may be related to the larger field of view (FOV) in the latter. The HTC Vive has a FOV of $110^{\circ}$. whereas the laptop screen in the desktop condition corresponded to a FOV of $23^{\circ}$. The increase in the advantage of eye-gaze over head-gaze with increasing field of view has already been demonstrated [Blattgerste et al. 2018]. However, their work did not study the impact on collaboration or teamwork.

Generally participants in Experiment 1 had more gaming experience than those in Experiment 2 and were all members of a specialist games design and programming course. Many thought that finding keys in the version of the game they played was easy. They were very used to game playing in a desktop environment. This may explain why the game durations in the desktop condition were lower than in the VR condition for both eye-gaze and head-gaze. The touchpad on the hand-held controller in the VR condition was less familiar than the keyboard and mouse to control movement, and this imposed a performance overhead in terms of time.

An important question is then why are the same results not as apparent in Experiment 2 when this was set up specifically to investigate the VR condition? The results from Experiment 2 also reflect the better performance with eye-gaze even though some were not significant. If only the second trials are considered (and the first trials are treated as practice), the mean duration of the eye-gaze trials was 226 seconds while the head-gaze trials had a mean duration of 322 seconds. In Experiment 1 in the VR condition only, the corresponding mean durations were 391 seconds and 513 seconds respectively. The rank sums of the teamwork scores in Experiment 2 on the second run were 135.5 for eye-gaze and 117.5 for head-gaze, and while not sufficiently different to be significant, the value for eye-gaze is higher than head-gaze.

There is also a difference in Experiment 2 that indicates teamwork performance with eye-gaze is better than with head-gaze (Figure 8). The two activities taken as indicators of collaboration showed increases in the proportion they took of the total trial duration when the second trial was eye-gaze. There were no similar increases when the second trial was head-gaze, suggesting that this is not simply an indicator of practice. It is entirely reasonable to question whether measures of time really reflect better performance

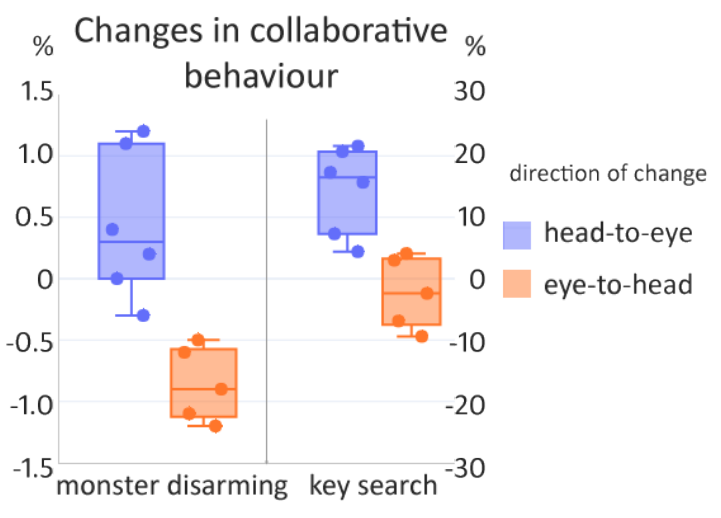

Figure 8: Change in collaboration rate for key search and monster disarming, separately for players moving from eyeto head-gaze condition and for player moving in the opposite way. Y-axis shows the proportion of game duration.

in a game. We have assumed that all players have wanted to complete the game as quickly as possible, and there was no indication during either experiment that players wanted to go off and explore parts of the house out of curiosity or fun. Also the participants' own assessment of teamwork was based on ratings of agreement with nine statements developed to assess collaborative work rather than collaborative games.

There is a good reason to try to assess the quality of collaboration from analysis of the communication between players during the game, as advocated by Seif El-Nasr et al. [Seif El-Nasr et al. 2010] rather than relying on post-hoc subjective assessment. This remains to be done.

Does eye-gaze really produce a better collaborative game experience and better teamwork than head-gaze in a VR environment? The experiments reported here strongly suggest that it does. It is not simply a question of social awareness of the other player. Headgaze provides that as well. This requires further investigation to answer properly.

\section{CONCLUSION}

We have shown that in the context of collaborative game play in a VR environment, eye-gaze leads to better performance and better teamwork than head-gaze. This conclusion is based on one game played for a short duration by pairs of players. The metrics used may be criticized that they do not adequately capture the richness of user experience with different types of collaborative games. Nevertheless, we have shown there to be a difference between the two modes which certainly merits further investigation to understand better what the advantages of eye-gaze are compared with headgaze in this context. This is particularly pertinent at a time when VR with head mounted displays looks set to expand enormously as a consumer product, and when eye-tracking looks set to become an integral standard component of these. 


\section{ACKNOWLEDGMENTS}

This research was funded by the Academy of Finland, project \#2501287895 Private and Shared Gaze: Enablers, Applications, Experiences $(\mathrm{GaSP})$.

\section{REFERENCES}

Richard Bates and Howell Istance. 2003. Why are eye mice unpopular? A detailed comparison of head and eye controlled assistive technology pointing devices. Universal Access in the Information Society 2, 3 (2003), 280-290.

Frank Biocca, Chad Harms, and Judee K Burgoon. 2003. Toward a more robust theory and measure of social presence: Review and suggested criteria. Presence: Teleoperators \& virtual environments 12, 5 (2003), 456-480.

Jonas Blattgerste, Patrick Renner, and Thies Pfeiffer. 2018. Advantages of Eye-gaze over Head-gaze-based Selection in Virtual and Augmented Reality Under Varying Field of Views. In Proceedings of the Workshop on Communication by Gaze Interaction (COGAIN '18). ACM, New York, NY, USA, Article 1, 9 pages. https://doi.org/10. 1145/3206343.3206349

Susan E Brennan, Xin Chen, Christopher A Dickinson, Mark B Neider, and Gregory J Zelinsky. 2008. Coordinating cognition: The costs and benefits of shared gaze during collaborative search. Cognition 106, 3 (2008), 1465-1477.

Guillaume Chanel, Mireille Bétrancourt, Thierry Pun, Donato Cereghetti, and Gaëlle Molinari. 2013. Assessment of computer-supported collaborative processes using interpersonal physiological and eye-movement coupling. In Humaine Association Conference on Affective Computing and Intelligent Interaction. IEEE, Geneva, Switzerland, 116âĂS122. https://doi.org/10.1109/ACII.2013.26

Inger Ekman, Guillaume Chanel, Simo Järvelä, J Matias Kivikangas, Mikko Salminen, and Niklas Ravaja. 2012. Social interaction in games: measuring physiological linkage and social presence. Simulation \& Gaming 43, 3 (2012), 321-338.

Kunal Gupta, Gun A Lee, and Mark Billinghurst. 2016. Do you see what I see? The effect of gaze tracking on task space remote collaboration. IEEE Transactions on Visualization and Computer Graphics 22, 11 (2016), 2413-2422.

Raija Hamalainen. 2008. Designing and evaluating collaboration in a virtual game environment for vocational learning. Computers \& Education 50, 1 (2008), 98-109.

Charlene Jennett, Anna L Cox, Paul Cairns, Samira Dhoparee, Andrew Epps, Tim Tijs, and Alison Walton. 2008. Measuring and defining the experience of immersion in games. International journal of human-computer studies 66, 9 (2008), 641-661.

Patrick Jermann, Marc-Antoine Nüssli, and Weifeng Li. 2010. Using dual eye-tracking to unveil coordination and expertise in collaborative Tetris. In Proceedings of the 24th BCS Interaction Specialist Group Conference. British Computer Society, Swinton, UK, UK, 36-44.

Thomas Küchelmann, Paola Torche, Manjunath Prasad, Kai Essig, and Thomas Schack. 2017. Automatic Analysis of Players Behavior in Real Dyadic Chess Situations. In 1st Workshop on âĂIJBehavior, Emotion and Representation: Building Blocks of Interactionâ $\breve{I}$. HAI, Bielefeld, Germany, 1-9.

Ayush Kumar, Michael Burch, and Klaus Mueller. 2018. Visual analysis of eye gazes to assist strategic planning in computer games. In Proceedings of the 3rd Workshop on Eye Tracking and Visualization. ACM, New York, NY, USA, 6.

Michael Lankes, Bernhard Maurer, and Barbara Stiglbauer. 2016. An Eye for an Eye Gaze Input in Competitive Online Games and Its Effects on Social Presence. In Proceedings of the 13th International Conference on Advances in Computer Entertainment Technology (ACE '16). ACM, New York, NY, USA, Article 17, 9 pages. https://doi.org/10.1145/3001773.3001774

Norman Murray, Dave Roberts, Anthony Steed, Paul Sharkey, Paul Dickerson, and John Rae. 2007. An assessment of eye-gaze potential within immersive virtual environments. ACM Transactions on Multimedia Computing, Communications, and Applications (TOMM) 3, 4 (2007), 8.

Abraham Naftali Oppenheim. 2000. Questionnaire design, interviewing and attitude measurement. Bloomsbury Publishing, New York, NY, USA.

Mary E Putt and Vernon M Chinchilli. 2004. Nonparametric approaches to the analysis of crossover studies. Statist. Sci. 19, 4 (2004), 712-719.

Yuan Yuan Qian and Robert J. Teather. 2017. The eyes don't have it: An empirical comparison of head-based and eye-based selection in virtual reality. In Proceedings of the 5th Symposium on Spatial User Interaction (SUI '17). ACM, New York, NY, USA, 91-98. https://doi.org/10.1145/3131277.3132182

Magy Seif El-Nasr, Bardia Aghabeigi, David Milam, Mona Erfani, Beth Lameman, Hamid Maygoli, and Sang Mah. 2010. Understanding and Evaluating Cooperative Games. In Proceedings of the SIGCHI Conference on Human Factors in Computing Systems (CHI '10). ACM, New York, NY, USA, 253-262. https://doi.org/10.1145/ 1753326.1753363

Harri Siirtola, Oleg Špakov, Howell Istance, and Kari-Jouko Räihä. 2019. Shared gaze in collaborative visual search. International fournal of Human-Computer Interaction 0, 0 (feb 2019), 1-13. https://doi.org/10.1080/10447318.2019.1565746 